\title{
Characteristic Analysis of Hierarchical and Non- Hierarchical Modulation for DVB-H
}

\author{
Shweta Shah \\ Assistant Professor \\ Electronics Department \\ S.V. National Institute of Tech., Surat, Gujarat, \\ India.
}

\begin{abstract}
DVB-H technology is based on ETSI standard designed to bring the broadcast services to battery-powered handheld receivers such as mobile. The system is defined based on the existing DVB-T (Terrestrial) system allowing for lesser power utilization, poorer signal power and speedy movements. DVB-H system uses OFDM technique to transport multimedia services in any of the three bandwidth modes i.e. 6, 7 and $8 \mathrm{MHz}$. In this paper, DVB-H physical layer is simulated using 4096 FFT mode and images are transmitted and received under AWGN channel. The main importance is on mapping methods, concentrating mainly on Hierarchical and Non-Hierarchical mapping methodologies. Bit error rate is analyzed for different code rates in both the type of methods and lastly concluded.
\end{abstract}

\section{Keywords}

BER; DVB-H; Hierarchical Modulation; simulation

\section{INTRODUCTION}

Today's era is surrounded by expertise and intelligent devices. New technologies, products and Services are constantly being evolved. The evolution of terrestrial broadcasting where the variety of ways in which TV is experienced is escalating dramatically. The new world of multimedia is an extension of digital multimedia delivered today via cable, satellite, DTH platforms, but with advanced compression and broadcasting technologies. The cellular world is also fairly diverse carrying smaller displays and requiring lower data rates to reproduce the information, but in a much more demanding situation of portable or mobile devices and varying signal strengths. The broadcast technologies that have allowed us to watch digitized TV at homes, have now been modified to enable the same programs to be broadcast to the mobiles [1]. Broadcasting to Handhelds is likely to be perceived as "TV on a Mobile" [2]

Among the conventional mapping schemes, Hierarchical Modulation (HM) is one of the strongest evaluations in DVB standard. The reception of multimedia content on mobile as well as coverage and bitrate are always a challenging task. HM provides the multiplexing of two information streams; here say two different images, in the same bandwidth with good coverage compared to conventional mapping schemes at the cost of increase in SNR. Section 2 provides the DVB-H physical layer description, section 3 covers the concept behind the HM, section 4 contains the simulation parameter and results and lastly conclusion is discussed.

\section{DVB - H Physical Layer}

One of the most interesting characteristics of the DVB-H standard $[8,9]$ is the ability to build hierarchical networks. Block diagram of DVB-H physical layer with HM is shown in figure 1:

\author{
U. D. Dalal, PhD. \\ Associate Professor \\ Electronics Department \\ S.V. National Institute of Tech., Surat, Gujarat, \\ India.
}

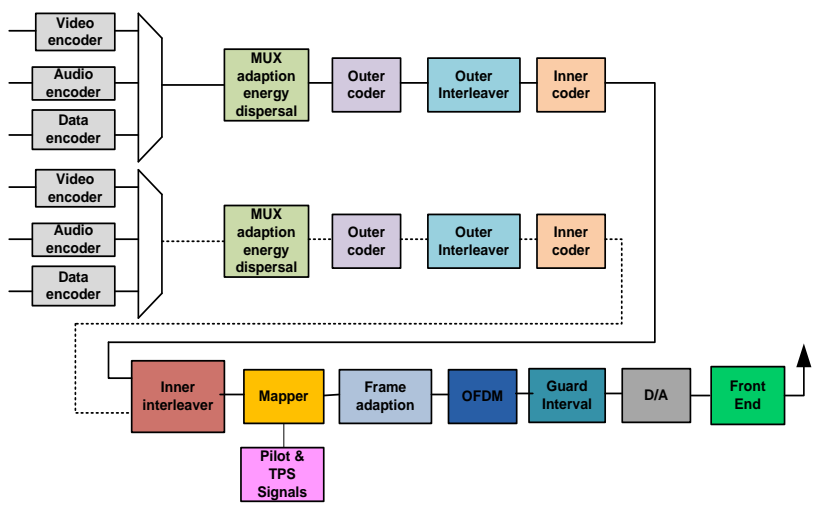

Fig 1 Block Diagram of a DVB-H System with Hierarchical Modulation

The input to the system can be any multimedia data. If HM (dotted line potion) is used than information coming from two channels can be different or same multiplexed at the stage of inner interleaver. These streams are initially given to energy dispersal which spread out the energy of information using shift register which are connected in the logic of $1+X^{14}+X^{15}$. An outer coder (Reed Solomon) of block length 204 bytes is used to provide first level of security. An outer interleaver followed by outer coder is used with interleaving depth of $\mathrm{I}=$ 12 as per the standard specification. An inner coder gives second level of security with variety of code rate support like $1 / 2,2 / 3,3 / 4,5 / 6$ and $7 / 8$. The selection of code rate affects the data rate value and the error correction capability. For HP (High priority) stream, 1/2 or $2 / 3$ code rates are used which provides maximum error protection and lower data rate while, for LP (Low Priority) stream other than these code rates like $2 / 3,3 / 4,5 / 6$ or $7 / 8$ can be applied. Code rate $7 / 8$ doesn't provide acceptable results hence it is omitted in simulation.

The inner interleaving provides bit and symbol wise interleaving. For HM, the bit mapping is important at this stage and explained in next section. After the mapping the OFDM frame is formed by addition of known signals as pilots. DVB-H supports three kinds of pilots continual, scattered and Transport Parameter Signaling (TPS), all with their importance. The compilation of OFDM frame makes signal ready to transmit. Here for the sake of simplicity AWGN channel is assumed. At the receiver side the reverse process are carried out to retrieve the information back.

\section{Concept of Hierarchical Modulation}

The two transmission signals vary in their susceptibility to noise. The basic signal (or HP) is more robust, in other words, heavily protected against noise and interference, but cannot support a high data rate, whereas the incremental signal (or LP) is capable of handling a higher data rate with a 
compromise of much less robustness. As a result, the coverage of the service area differs in size. In practice, the basic signal can be used for portable indoor and mobile reception, while the low priority signal can be chosen to deliver a HDTV program to a permanent receiver, such as a $\mathrm{TV}$ at home. The total data rate of the two hierarchical streams is identical to that of a non-hierarchical modulation scheme. The net data rate will however be slightly lower because twice the MPEG-TS overhead is added in the HM, on expense of the two multiplexes [3].

The ability to use hierarchies in the physical layers gives flexibility to the network and service providers to plan their network. The hierarchies used in mappings are more popular and acceptable. In DVB-H, multiplexing of two information streams is carried out at the stage of inner interleaving. For example, the input, which consists of up to two bit streams, is separated into v sub-streams, where $\mathrm{v}=2$ for QPSK, $\mathrm{v}=4$ for 16-QAM, and $\mathrm{v}=6$ for $64-\mathrm{QAM}$. In non-hierarchical mode, the single input stream is separated into $\mathrm{v}$ sub-streams. In HM the HP stream is demultiplexed into two sub-streams and the LP stream is separated into v-2 sub-streams. This applies in both uniform and non-uniform QAM modes. The demultiplexer has to map the input bits xdi onto output bits be,do, following these rules. These will be observed in Table 1,2 \& 3clearly [4].

Table 1. QPSK demultiplexing for Non-Hierarchical / Hierarchical Transmission

QPSK Non-Hierarchical / Hierarchical Transmission: $x_{0}$ maps to $b_{0,0}$ $x_{I}$ maps to $b_{1,0}$

Table 2. 16QAM demultiplexing for Non-Hierarchical $\&$ Hierarchical Transmission

\begin{tabular}{|c|c|}
\hline $\begin{array}{c}\text { 16QAM Non-Hierarchical } \\
\text { Transmission: }\end{array}$ & $\begin{array}{c}\text { 16QAM Hierarchical } \\
\text { Transmission: }\end{array}$ \\
\hline$x_{0}$ maps to $b_{0,0}$ & $\boldsymbol{x}_{{ }_{0}}$ maps to $\boldsymbol{b}_{0,0}$ \\
\hline$x_{I}$ maps to $b_{2,0}$ & $\boldsymbol{x}_{{ }_{1}}$ maps to $\boldsymbol{b}_{1,0}$ \\
\hline$x_{2}$ maps to $b_{1,0}$ & $\boldsymbol{x}_{{ }_{1}{ }_{0} \text { maps to } \boldsymbol{b}_{2,0}}$ \\
\hline$x_{3}$ maps to $b_{3,0}$ & $\boldsymbol{x}_{{ }_{1}{ }_{1}}$ maps to $\boldsymbol{b}_{3,0}$ \\
\hline
\end{tabular}

The susceptibility to noise of the HP and the LP stream can be influenced by ratio $\alpha$ known as modulation parameter, which is defined by the minimum distances between quadrants $\mathrm{d} 1$ and between points $\mathrm{d} 2$ given by

$$
\alpha=\frac{d_{1}}{d_{2}}
$$

Table 3. 64QAM demultiplexing for Non-Hierarchical \& Hierarchical Transmission

\begin{tabular}{|c|c|}
\hline $\begin{array}{l}\text { 64QAM Non-Hierarchical } \\
\text { Transmission: }\end{array}$ & $\begin{array}{l}\text { 64QAM Hierarchical } \\
\text { Transmission: }\end{array}$ \\
\hline$x_{0}$ maps to $b_{0,0}$ & $x_{0}^{\prime}$ maps to $b_{0,0}$ \\
\hline$x_{1}$ maps to $b_{2,0}$ & $x_{1}^{\prime}$ maps to $b_{1,0}$ \\
\hline$x_{2}$ maps to $b_{4,0}$ & $x^{\prime \prime}{ }_{0}$ maps to $b_{2,0}$ \\
\hline$x_{3}$ maps to $b_{1,0}$ & $x^{\prime \prime}{ }_{1}$ maps to $b_{4,0}$ \\
\hline$x_{4}$ maps to $b_{3,0}$ & $x^{\prime \prime}{ }_{2}$ maps to $b_{3,0}$ \\
\hline$x_{5}$ maps to $b_{5,0}$ & $x^{\prime \prime}{ }_{3}$ maps to $b_{5,0}$ \\
\hline
\end{tabular}

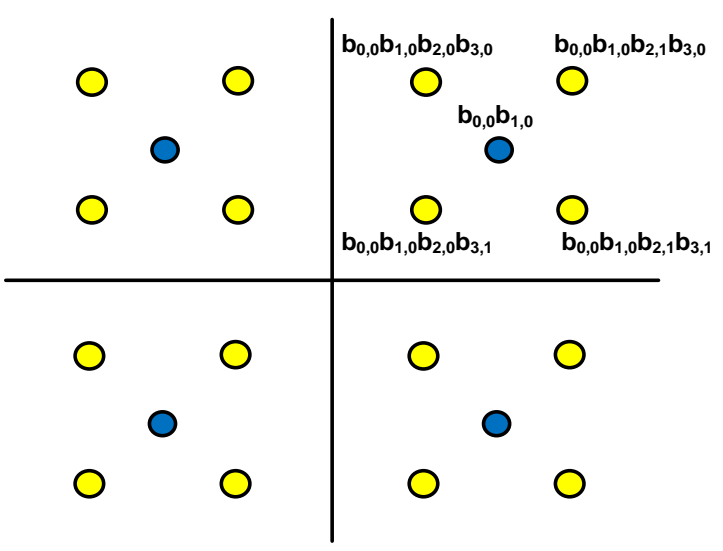

Fig 2 Mapping of I/P Bits onto O/P Modulation Symbols for Hierarchical 16QAM

With $\alpha=1$, the LP and HP stream show equal susceptibility to noise. With $\alpha>1$ (can be 2 or 4), the MSBs of the HP stream become more robust to channel noise due to the higher distance between the quarters $[5,6]$. In HM, the possible states are interpreted differently than in the non-hierarchical case. The location of a state within its quadrant is regarded as special information in HM.

Basically there are three parameters required to describe a HM scheme: $\alpha$, and the code rates for the HP and LP streams. It is possible to enlarge the coverage area of the HP stream even further by changing the modulation parameter $\alpha$ to 2 or 4 at the expense of the robustness of the LP stream. i.e., Increase of $\alpha$ leads to increase of the average transmission power. Here, one may not observe large SNR variation for HP stream but there will be noticeable increment in requirement of SNR by LP stream.

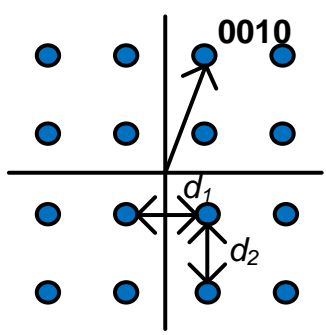

Fig 3 Basic Constellation 


\section{Simulation Parameter \& Results}

For the simulation MATLAB code is developed and the parameter for simulation is mentioned in table 4 . The input to the system is two gray images as shown in fig. 4 (a) and the received image for $\mathrm{SNR}=5 \mathrm{~dB}$ is shown in fig. $4(\mathrm{~b})$.

Table 4. DVB-H specification for Simulation

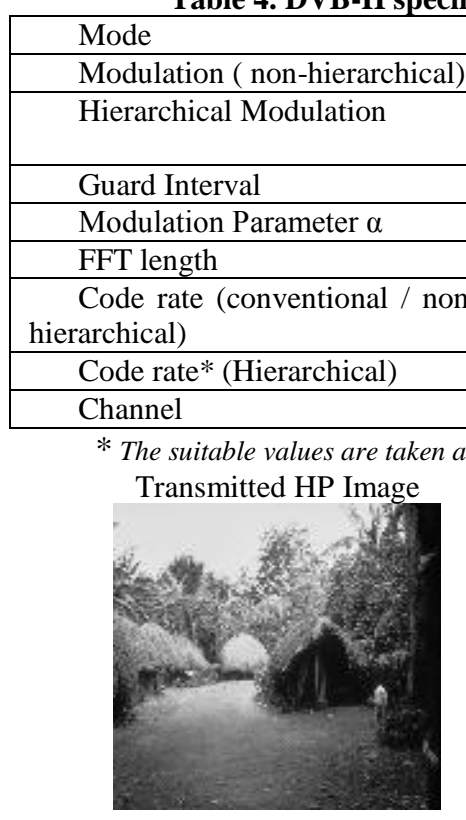
$4 \mathrm{k}$

\begin{tabular}{|l|l|}
\hline 16QAM, 64QAM \\
\hline $\begin{array}{c}\text { QPSK in 16QAM, } \\
\text { QPSK in 64QAM }\end{array}$ \\
\hline $1 / 4$ \\
\hline $1,2,4$ \\
\hline - & $3 / 4,5 / 6$ \\
\hline & $1 / 2(\mathrm{HP}), 3 / 4,5 / 6$ (LP) \\
\hline
\end{tabular}

* The suitable values are taken as per the standard document.
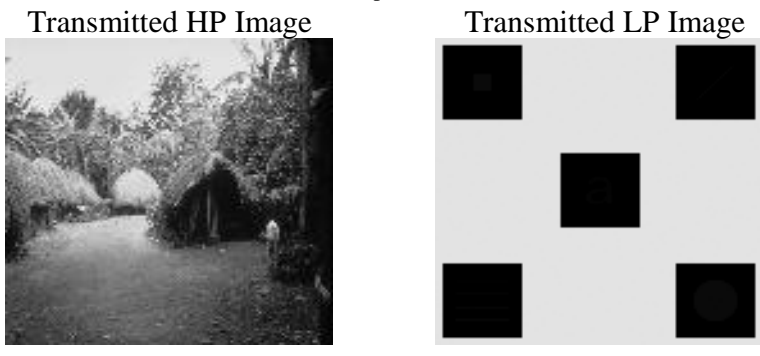

(a)

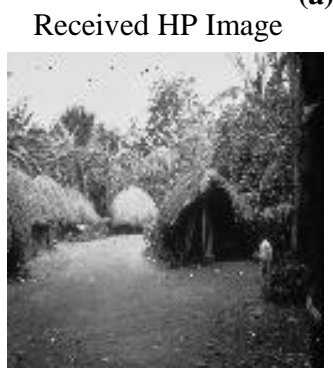

Received LP Image

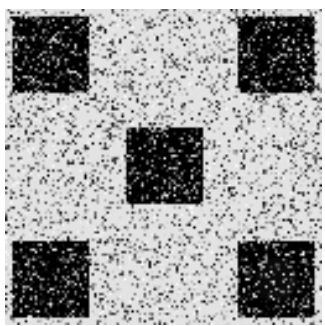

(b)

Fig 4 (a) Transmitted HP and LP images, (b) Received HP and $L P$ images at $S N R=5 \mathrm{~dB}$

The resultant graph shown in fig. $5,6,7 \& 8$ proves the importance of HM compared to conventional mapping method 16QAM. The simulation results shows the application of different code rates affects the SNR values as well as with the little increase in SNR also gives better coverage [7]. Application of HM avoids fundamental drawback of conventional modulation of abrupt breakdown in reception below a critical field strength level in digital transmission.

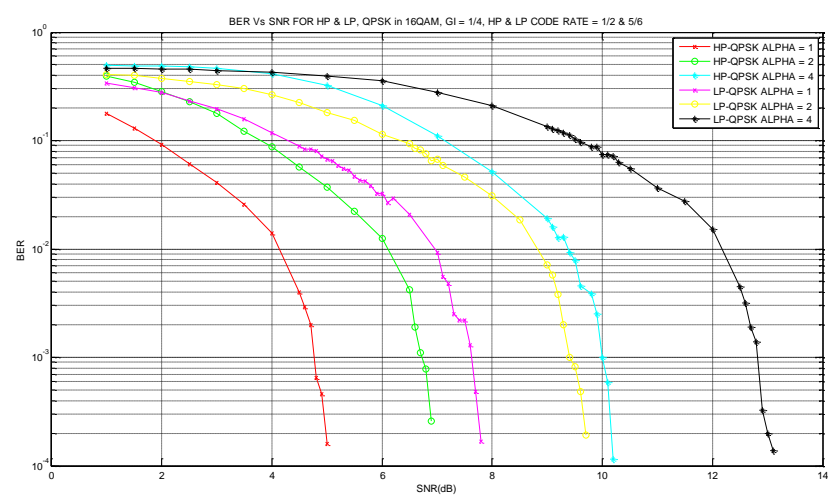

Fig 5 SNR Vs BER for HP, LP \& 16QAM, code rate $=1 / 2$ (HP), 3/4 (LP \& QPSK)

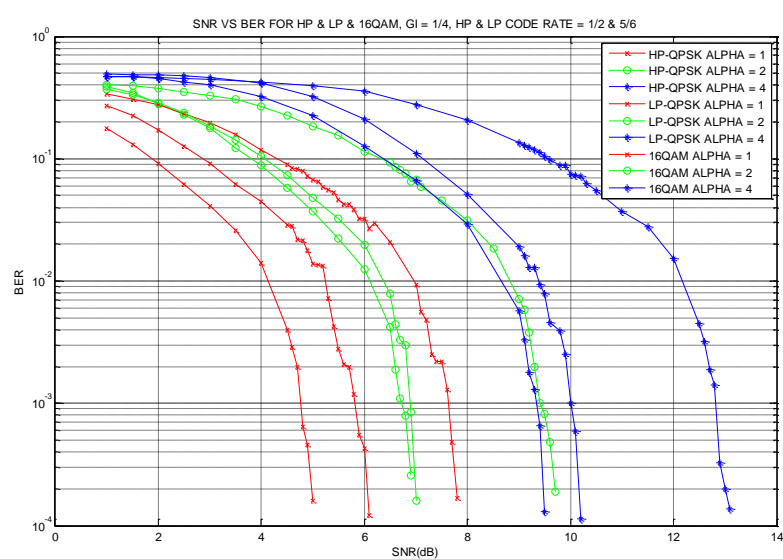

Fig 6 SNR Vs BER for HP, LP \& 16QAM, code rate $=1 / 2$ (HP), 5/6 (LP \& QPSK)

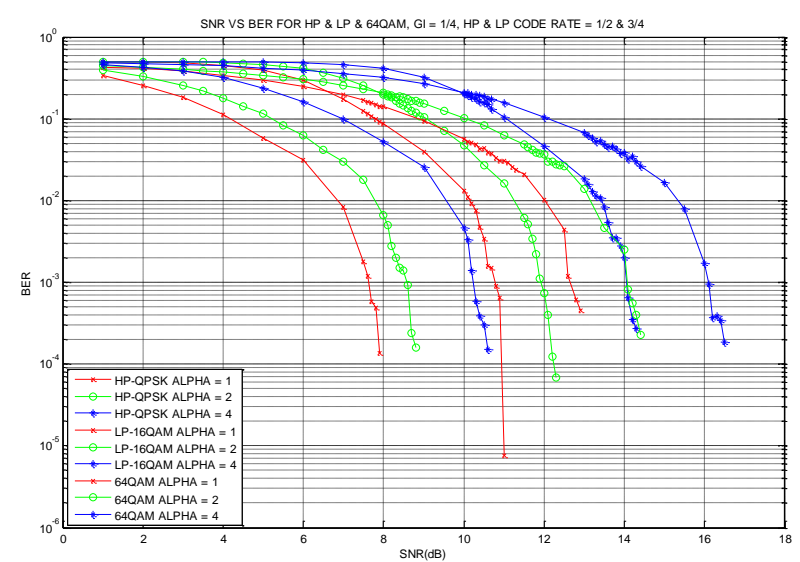

Fig 7 SNR Vs BER for HP, LP \& 64QAM, code rate $=1 / 2$ (HP), 3/4 (LP \& 16QAM) 


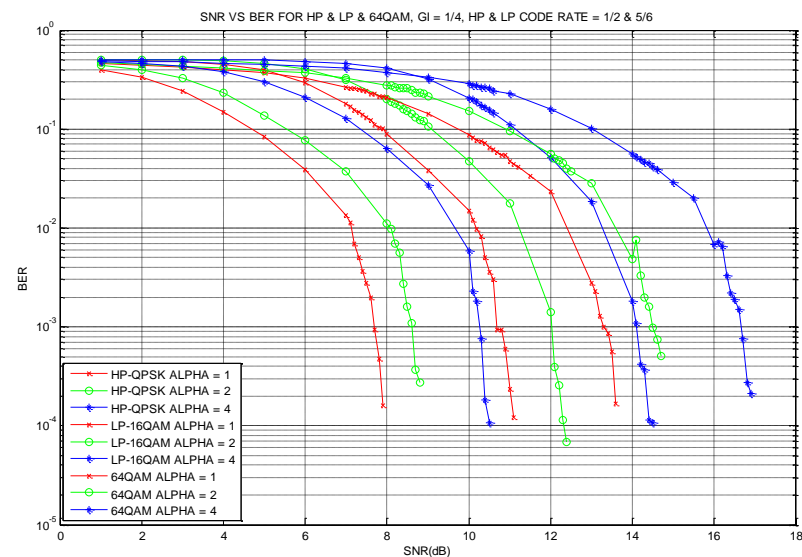

Fig 8 SNR Vs BER for HP, LP \& 64QAM, code rate $=1 / 2$ (HP), 5/6 (LP \& 16QAM)

\section{Conclusion}

The conclusion is that, in certain practical cases, HM can represent a better alternative than non-HM. It provides two autonomous or dependent multiplexes (HP and LP) over a single TV frequency channel. The HP stream is dedicated to receivers with challenging channel superiority, unlike the LP stream which requires a good SNR to be decoded. A portion of the HP data rate can be used in particular for portable indoor and movable reception. With this arrangement, very flexible service coverage concepts can be developed for future requirements. Also increment in Modulation parameter increases average transmission power which support larger coverage area.

\section{REFERENCES}

[1] A. Kumar, Mobile TV DVB-H, DMB, 3G Systems and Rich Media Applications, Burlington, MA: Focal Press, 2007.

[2] J. T. J. Penttinen et al., "The DVB-H Handbook: The Functioning and Planning of Mobile TV". Chichester, UK: Wiley, 2009.

[3] Floriano De Rango, Digital Video, INTECH, Croatia, February 2010.

[4] ETSI EN 300744 V1.6.1 (2008-09). "European Standard. Digital Video Broadcasting (DVB); Framing structure, channel coding and modulation for DTT".

[5] Cornelius Hellge, Shpend Mirta, Thomas Schierl, and Thomas Wiegand, " Mobile TV with SVC and Hierarchical Modulation for DVB-H Broadcast Services ", November 3, 2008.

[6] Hugo M'ericy, J. Lacany, F. Arnalz, G. Lesthieventx, M. Boucheret, "Improving Broadcast Channel Rate Using Hierarchical Modulation", 17 Jan 2012. Available at arXiv:1111.2425v2 [cs.NI]

[7] S. M. Sadough, Y. Pierre Duhamel, On the Interaction between Channel Coding and Hierarchical Modulation, IEEE International Conference on Communications Society proceedings, pp.1-5, 2009.

[8] ETSI, EN 302304 v1.1.1, Digital Video Broadcasting (DVB); Transmission System for Handheld Terminals (DVB-H), October, 2004.

[9] M. Kornfeld and U. Reimers, DVB-H, The Emerging Standard for Mobile Data Communication, EBU Technical Review, January, 2005. 